\title{
Immune checkpoint inhibitors in esophagogastric adenocarcinoma: do the results justify the hype?
}

\author{
Megan Greally, Geoffrey Y. Ku \\ Gastrointestinal Oncology Service, Department of Medicine, Memorial Sloan Kettering Cancer Center, New York, USA \\ Correspondence to: Megan Greally. Gastrointestinal Oncology, Department of Medicine, Memorial Sloan Kettering Cancer Center, 1275 York Avenue, \\ New York, USA. Email: greallym@mskcc.org. \\ Comment on: Janjigian YY, Bendell J, Calvo E, et al. CheckMate-032 Study: Efficacy and Safety of Nivolumab and Nivolumab Plus Ipilimumab in \\ Patients With Metastatic Esophagogastric Cancer. J Clin Oncol 2018;36:2836-44.
}

Submitted Nov 21, 2018. Accepted for publication Nov 29, 2018.

doi: $10.21037 /$ jtd.2018.12.01

View this article at: http://dx.doi.org/10.21037/jtd.2018.12.01

Immune checkpoint inhibitors have transformed treatment paradigms in several cancers, many of which were previously associated with a poor prognosis, including melanoma and lung cancer. Given the poor outcomes from treatment with chemotherapy and targeted therapies in this disease, there has been strong interest in the evaluation of immunotherapy.

The ATTRACTION-2 (nivolumab) and KEYNOTE-059 (pembrolizumab) studies confirmed the activity of anti-programmed death (PD)-1 antibodies in the chemorefractory setting $(1,2)$ and in a landmark approval, pembrolizumab was approved in the US in September 2017 for patients with advanced gastric and gastroesophageal junction (GEJ) adenocarcinoma whose tumors express PD-L 1 and who have received $\geq 2$ chemotherapy regimens. Nivolumab is approved in Japan, regardless of PD-L1 status. While approval of these drugs represents encouraging progress in a disease with a dismal prognosis, benefit is modest with single-agent therapy in an unselected population. Many questions remain regarding patient selection, treatment timing, combinatorial strategies and appropriate biomarkers.

In August 2018, Janjigian and colleagues reported results from the Checkmate-032 trial in the Fournal of Clinical Oncology (3). This was an open-label, phase I/II, multicohort study which evaluated the safety and activity of nivolumab alone or in combination with ipilimumab, in two dosing schedules, in patients with advanced chemorefractory gastric, esophageal or GEJ adenocarcinoma. One-hundred sixty patients were enrolled sequentially to 3 different cohorts: $3 \mathrm{mg} / \mathrm{kg}$ nivolumab every 2 weeks (NIVO3), $1 \mathrm{mg} / \mathrm{kg}$ of nivolumab plus $3 \mathrm{mg} / \mathrm{kg}$ of ipilimumab (NIVO1 + IPI3) or $3 \mathrm{mg} / \mathrm{kg}$ nivolumab plus $1 \mathrm{mg} / \mathrm{kg}$ of ipilimumab (NIVO3 + IPI1) every 3 weeks for 4 cycles followed by nivolumab $3 \mathrm{mg} / \mathrm{kg}$ every 2 weeks until disease progression or intolerable toxicity. The primary endpoint was objective response rate (ORR). While PD-L1 positivity was assessed using a cut-off of $\geq 1 \%$ tumor staining on immunohistochemistry (IHC) (assessed by the Dako 28-8 pharmDx assay), patients were not required to have PD-L1 positive tumors to receive therapy.

All patients had good ECOG performance status (0 or 1), primary tumor location was gastric or GEJ in $84 \%$ and $79 \%$ of patients had received $\geq 2$ prior lines of therapy. Regarding PD-L1 status, $38 \%, 24 \%$ and $30 \%$ of evaluable patients were PD-L1 positive in the NIVO3, NIVO1 + IPI3 and NIVO3 + IPI1 cohorts respectively. Results from 59 patients in the NIVO3 cohort suggest similar activity to that observed in previous studies. The highest ORR of all 3 cohorts was $24 \%$, in the NIVO1 + IPI3 group. Detailed efficacy results are outlined in Table 1.

Any increased activity in the NIVO1 + IPI 3 arm was counterbalanced by the fact that the highest rate of grade $\geq 3$ toxicity occurred in this arm (47\%) and treatment-related adverse events resulted in treatment discontinuation in $20 \%$ of this patient group. In contrast, the grade $\geq 3$ toxicity rate in the NIVO3 arm was $17 \%$.

While the results of this study should be interpreted with caution given the relatively small numbers enrolled in each cohort and because patients were assigned to cohorts 
Table 1 Response and survival data in each cohort

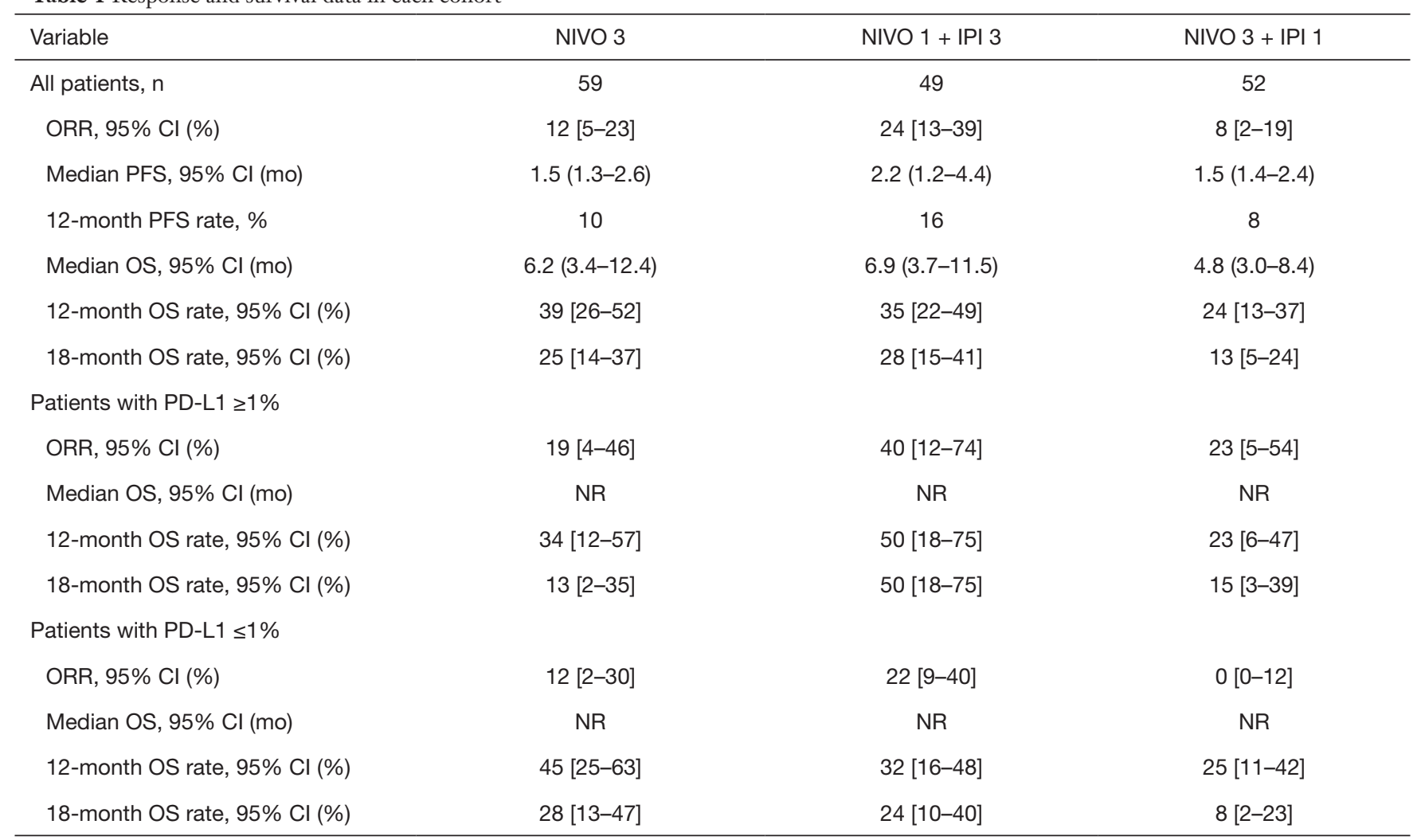

NIVO 3, nivolumab 3 mg/kg; NIVO 1, nivolumab 1 mg/kg; IPI3, ipilimumab 3 mg/kg; IPI 1, ipilimumab 1 mg/kg; ORR, objective response rate; OS, overall survival; $\mathrm{Cl}$, confidence interval; NR, not reported.

sequentially in a non-randomized fashion, there are several important observations. Firstly, the ORR and progressionfree survival results observed between ATTRACTION-2, KEYNOTE-059 and the NIVO3 cohort of Checkmate-032 were virtually identical, confirming consistent benefit in Western and Asian populations $(1,2)$. Secondly, the addition of low dose ipilimumab to full dose nivolumab in the NIVO3 + IPI1 arm did not seem to improve the ORR compared to nivolumab alone. Finally, an impressive $40 \%$ ORR was observed in PD-L1+ patients in the NIVO1 + IPI3 cohort, the highest ORR reported to date with immunotherapy in esophagogastric cancer.

However, median, 12- and 18-month overall survival (OS) rates were similar between the NIVO3 and the NIVO1 + IPI3 groups despite a numerically higher ORR with combination therapy. The authors acknowledge that this may be partly explained by the substantially higher number of patients with microsatellite unstable (MSI) tumors in the NIVO3 group than the NIVO1 + IPI3 group ( $28 \%$ vs. $9 \%$ ). This remarkably high proportion of patients with MSI tumors may also explain the observation that the 12 -month OS rate in the NIVO3 arm (39\%) is numerically superior to the 12 -month OS rates of $26.2 \%$ and $23.4 \%$ in ATTRACTION-2 and KEYNOTE-059 respectively. The high incidence of MSI tumors in the study exceeds the much lower incidence $(<5 \%)$ in other larger data sets (2) and suggests that patients may have been preferentially referred for the Checkmate-032 study; this potential bias raises questions about the generalizability about the study findings.

Indeed, MSI status may have impacted overall results in previously reported studies. For example, $4 \%$ of patients who underwent MSI testing in KEYNOTE-059 were MSI (2). The ORR in this group was $57.1 \%$. When these patients were excluded from the analysis, the ORR was $9 \%$ in the microsatellite stable (MSS) population; the ORR in the intention-to-treat (ITT) population was $11.6 \%$. As the 12-month OS rates for the MSI, MSS and ITT populations were not reported, one can only speculate to what extent the MSI population is driving the long-term survival results 
across these studies of single-agent immune checkpoint inhibitors in the chemorefractory setting.

While associated with additional toxicity, the ORR seen with NIVO1 + IPI3 led to this dose being selected for further evaluation in the phase III Checkmate-649 study (NCT02872116), which randomizes patients to FOLFOX, FOLFOX plus nivolumab or ipilimumab/nivolumab. However, the ipilimumab/nivolumab arm is now closed after an interim analysis and accrual to the remaining arms continues. Whether the premature closure is because of increased toxicity and/or decreased efficacy relative to the chemotherapy-containing arms remains to be seen. While disappointing, this development is not entirely surprising given the known toxicity of the NIVO1 + IPI3 combination and suggest that it is unlikely that combination ipilimumab/ nivolumab will have a frontline role in esophagogastric cancer.

Evaluation of immunotherapeutic strategies continues at pace in esophagogastric cancer. Recently, results from two phase III trials evaluating anti-PD-1/PD-L1 antibodies $v s$. chemotherapy were reported. The JAVELIN 300 trial randomized patients previously treated with $\geq 2$ lines of chemotherapy to avelumab or physician's choice paclitaxel or irinotecan and found no improvement in OS in avelumab-treated patients (4). Furthermore, there was no improvement in OS in patients who were PD-L1 positive as defined by $\geq 1 \%$ of tumor cells. This suggests that singleagent anti-PD-1/PD-L1 therapy, which has only previously been studied in single-arm studies or against placebo, may be inferior to chemotherapy in the $3^{\text {rd }}$-line setting.

Similarly, KEYNOTE-061 also failed to show a survival benefit for pembrolizumab over paclitaxel in the secondline setting in patients with PD-L1 CPS (combined positive score; a proportional assessment of PD-L1 staining on tumor and immune cells) expression $>1$ (5). Of note, in a post-hoc analysis, patients with CPS scores $\geq 5$ and $\geq 10$ did appear to obtain benefit. These disappointing results add weight to the hypothesis that only a minority of patients benefit from single-agent immunotherapy. Most immunotherapy-treated patients experienced rapid progression in these studies, with a median progression-free survival of less than 2 months.

These two studies also show variable results when patients were stratified by PD-L1 status. In the JAVELIN study, results were not altered when patients were stratified by PD-L1 status (additional analysis of PD-L1 assessment in tumor and immune cells showed similar results; data not reported) similar to ATTRACTION-2 and the NIVO3 arm of Checkmate-032 where the same methodology for PDL1 assessment was utilized (tumor proportion score; TPS) $(1,3,4)$. In contrast, PD-L1 was assessed using the IHC 22C3 pharmDx (Dako) test by CPS in KEYNOTE-059 and KEYNOTE-061 and the ORR was higher in pembrolizumab-treated patients who were PD-L1 CPS $\geq 1$ and CPS $\geq 10$ in KEYNOTE-059 and KEYNOTE-061 respectively $(2,5)$.

Finally, in a recent press release it was reported that in the phase III KEYNOTE-181 study evaluating pembrolizumab $v s$. physician's choice chemotherapy in patients with advanced esophageal/GEJ adenocarcinoma and squamous cell carcinoma who had progressed after first-line therapy and whose tumors expressed PD-L1 CPS $\geq 10$, pembrolizumab met the primary endpoint of improved OS (NCT02564263). Detailed results are awaited.

These contradictory data highlight the need for further refinement of PD-L1 testing methodology and optimal cutoffs. The results outlined above suggest that CPS is a more suitable biomarker than TPS and the consistent results across KEYNOTE 061 and KEYNOTE 181 suggest that a higher CPS than $\geq 1$ may be appropriate for stratifying patients going forward. An important observation regarding the KEYNOTE-061 and KEYNOTE-181 is that the comparator arm in both studies was single agent chemotherapy with a taxane or irinotecan. However, the standard second-line treatment is now paclitaxel with ramucirumab, the anti-vascular endothelial growth factor receptor-2 antibody, based on the RAINBOW trial, which showed higher ORR and OS compared to paclitaxel alone (6). Therefore, it remains unclear whether single agent immune checkpoint inhibitors would be superior to combination therapy with paclitaxel/ramucirumab in the second-line setting.

The issues with PD-L1 discussed above underline the need to identify novel biomarkers to allow us to better identify patients likely to obtain prolonged disease control from anti-PD-1/PD-L1 monotherapy. In KEYNOTE-059, an 18-gene T-cell inflamed gene expression signature was associated with improved response to pembrolizumab (2). Elevated tumor mutation burden has been shown to predict response to checkpoint inhibitors across several tumor types (7-9). Prospective studies are required to determine if this could be a useful biomarker in this setting. Knowledge of The Cancer Genome Atlas (TCGA) may be helpful in improving outcomes in the future (10). The EBV and MSI subtypes have elevated mutation burden and are more likely to respond to single-agent checkpoint inhibitors. In 
a recent study, the ORR to pembrolizumab was $100 \%$ in EBV and MSI-high patients and only $12 \%$ and $5 \%$ in the genomically stable (GS) and chromosomal instability (CIN) subtypes (11). Combinatorial strategies should be evaluated in the GS and CIN subtype patients in an effort to improve these modest response rates.

The results of Checkmate-032 build on data from the ATTRACTION-2 and KEYNOTE-059 and provide an important foundation for continued evaluation of immunotherapeutic strategies in esophagogastric cancer which may further expand the role of immune checkpoint inhibitors in this disease. However, single-agent activity of a PD-1 inhibitor remains modest and the toxicities of ipilimumab/nivolumab make it unlikely this combination will be advanced further, given the premature closure of this arm in the first-line Checkmate 649 study.

Thus, the focus has turned to novel combinatorial strategies, incorporation of these drugs earlier in treatment paradigms and identification of more optimal biomarkers in an effort to exploit the potential of these agents in this disease. There are now numerous accrued, ongoing or planned studies investigating immune checkpoint inhibitors in combination with chemotherapy in the first-line setting [KEYNOTE-062 randomized patients to pembrolizumab monotherapy or in combination with cisplatin/ fluoropyrimidine $v s$. chemotherapy alone (NCT02494583) and Checkmate-649 is comparing nivolumab plus fluoropyrimidine/oxaliplatin to chemotherapy alone (NCT02872116)], other immunotherapies and targeted therapies such as trastuzumab (NCT03615326) and ramucirumab (NCT02999295). For example, two studies that are rapidly evaluating novel combinatorial strategies are the FRACTION (Fast Real-time Assessment of Combination Therapies in Immuno-Oncology study)Gastric Cancer adaptive design phase II study and the MORPHEUS-Gastric Cancer study (NCT02935634 and NCT03281369 respectively). It is hoped that these novel doublets may be less toxic than ipilimumab/nivolumab.

Finally, immune checkpoint inhibitors are now also being evaluated in earlier stage disease; the phase III Checkmate-577 (NCT02743494) is a global phase III study evaluating adjuvant nivolumab $v$ s. placebo in patients with locally advanced GEJ carcinoma who have persistent disease following pre-operative chemoradiation and surgery with clear margins and the phase III KEYNOTE-585 study (NCT03221426) is investigating the addition of pembrolizumab to peri-operative chemotherapy in patients with gastric or GEJ adenocarcinoma. Several studies are also adding immunotherapy to pre-operative chemoradiation for esophageal or GEJ cancer, including 2 studies that involve our group [one with the anti-PD-L1 antibody durvalumab in adenocarcinoma tumors (NCT02962063) and the other with nivolumab for squamous cell cancers (NCT03278626)]. Ongoing evolution of these studies, along with data from correlative studies, may allow us to gain a more nuanced understanding of the clinical and molecular characteristics which may predict responses to these therapies going forward.

\section{Acknowledgements}

None.

\section{Footnote}

Conflicts of Interest: GY Ku reports research grants from Aduro Biotech, Arog Pharmaceuticals, Astra-Zeneca, Bristol-Myers Squibb, Merck and Pieris; and consulting for Bristol-Myers Squibb, Eli Lilly, Merck and Pieris. The other author has no conflicts of interest to declare.

\section{References}

1. Kang YK, Boku N, Satoh T, et al. Nivolumab in patients with advanced gastric or gastro-oesophageal junction cancer refractory to, or intolerant of, at least two previous chemotherapy regimens (ONO-4538-12, ATTRACTION-2): a randomised, double-blind, placebocontrolled, phase 3 trial. Lancet 2017;390:2461-71.

2. Fuchs CS, Doi T, Jang RW, et al. Safety and Efficacy of Pembrolizumab Monotherapy in Patients With Previously Treated Advanced Gastric and Gastroesophageal Junction Cancer: Phase 2 Clinical KEYNOTE-059 Trial. JAMA Oncol 2018;4:e180013.

3. Janjigian YY, Bendell J, Calvo E, et al. CheckMate-032 Study: Efficacy and Safety of Nivolumab and Nivolumab Plus Ipilimumab in Patients With Metastatic Esophagogastric Cancer. J Clin Oncol 2018;36:2836-44.

4. Bang YJ, Ruiz EY, Van Cutsem E, et al. Phase III, randomised trial of avelumab versus physician's choice of chemotherapy as third-line treatment of patients with advanced gastric or gastro-oesophageal junction cancer: primary analysis of JAVELIN Gastric 300. Ann Oncol 2018;29:2052-60.

5. Shitara K, Özgüroğlu M, Bang YJ, et al. Pembrolizumab versus paclitaxel for previously treated, advanced gastric or 
gastro-oesophageal junction cancer (KEYNOTE-061): a randomised, open-label, controlled, phase 3 trial. Lancet 2018;392:123-33.

6. Wilke H, Muro K, Van Cutsem E, et al. Ramucirumab plus paclitaxel versus placebo plus paclitaxel in patients with previously treated advanced gastric or gastro-oesophageal junction adenocarcinoma (RAINBOW): a double-blind, randomised phase 3 trial. Lancet Oncol 2014;15:1224-35.

7. Rosenberg JE, Petrylak DP, Heijden MSVD, et al. PDL1 expression, Cancer Genome Atlas (TCGA) subtype, and mutational load as independent predictors of response to atezolizumab (atezo) in metastatic urothelial carcinoma (mUC; IMvigor210). J Clin Oncol 2016;34:104.

8. Hellmann MD, Ciuleanu TE, Pluzanski A, et al.

Cite this article as: Greally M, Ku GY. Immune checkpoint inhibitors in esophagogastric adenocarcinoma: do the results justify the hype? J Thorac Dis 2018;10(12):6407-6411. doi: 10.21037/jtd.2018.12.01
Nivolumab plus Ipilimumab in Lung Cancer with a High Tumor Mutational Burden. N Engl J Med 2018;378:2093-104.

9. Hellmann MD, Callahan MK, Awad MM, et al. Tumor Mutational Burden and Efficacy of Nivolumab Monotherapy and in Combination with Ipilimumab in Small-Cell Lung Cancer. Cancer Cell 2018;33:853-861.e4.

10. Cancer Genome Atlas Research Network. Comprehensive molecular characterization of gastric adenocarcinoma. Nature 2014;513:202-9.

11. Kim ST, Cristescu R, Bass AJ, et al. Comprehensive molecular characterization of clinical responses to PD-1 inhibition in metastatic gastric cancer. Nat Med 2018;24:1449-58. 